\title{
Postoperative morbidity and health-related quality of life in children with delayed reconstruction of esophageal atresia: a nationwide Swedish study
}

Michaela Dellenmark-Blom ( $\sim$ michaela.dellenmark.blom@gu.se)

University of Gothenburg Institute of Clinical Sciences: Goteborgs universitet Institutionen for kliniska vetenskaper https://orcid.org/0000-0002-1467-9427

Sofie Örnö Ax

Queen Silvia Children's Hospital: Sahlgrenska universitetssjukhuset Drottning Silvias barn- och ungdomssjukhus

Elin Öst

Department of Pediatric Surgery, Karolinska University Hospital, Stockholm, Sweden

Jan F Svensson

Department of Pediatric Surgery, Karolinska University Hospital, Stockholm, Sweden

Ann-Marie Kassa

Department of Pediatric Surgery, University Children's Hospital, Uppsala, Sweden

Linus Jönsson

Department of Pediatric Surgery, Queen Silvia Children's Hospital, Gothenburg, Sweden

Kate Abrahamsson

Department of Pediatric Surgery, Queen Silvia Children's Hospital, Gothenburg, Sweden

Vladimir Gatzinsky

Department of Pediatric Surgery, Queen Silvia Children's Hospital, Gothenburg, Sweden

Pernilla Stenström

Department of Pediatric Surgery, Skane University Hospital, Lund, Sweden

AnnaMaria Tollne

Department of Pediatric Surgery, Karolinska University Hospital, Stockholm, Sweden

Erik Omling

Department of Pediatric Surgery, Skane University Hospital, Lund, Sweden

Helene Engstrand Lilja

Department of Pediatric Surgery, University Children's Hospital, Uppsala, Sweden

\section{Research Article}

Keywords: esophageal atresia, long-gap esophageal atresia, health-related quality of life, delayed reconstruction, long-term morbidity, postoperative outcomes

Posted Date: January 31st, 2022

DOI: https://doi.org/10.21203/rs.3.rs-1291395/v1

License: @ (i) This work is licensed under a Creative Commons Attribution 4.0 International License. Read Full License 


\section{Abstract}

Background In 10-15\% of children with esophageal atresia (EA) delayed reconstruction of esophageal atresia (DREA) is necessary due to long-gap EA and/or prematurity/low birth weight. They represent a patient subgroup with high risk of complications. We aimed to evaluate postoperative morbidity and healthrelated quality of life (HRQOL) in a Swedish national cohort of children with DREA.

Methods Postoperative morbidity, age-specific generic HRQOL (PedsQL ${ }^{T M} 4.0$ ) and condition-specific HRQOL (The EA-QOL questionnaires) in children with DREA were compared with children with EA who had primary anastomosis (PA). Factors associated with the DREA group's HRQOL scores were analyzed using Mann-Whitney U-test and Spearman's rho. Clinical data was extracted from the medical records. Significance level was $p<0.05$.

Results Thirty-four out of 45 families of children with DREA were included and 30 returned the questionnaires( $n=8$ children aged $2-7$ years; $n=22$ children aged 8-18 years). Compared to children with PA(42 children aged 2-7 years; 64 children aged 8-18 years), there were no significant differences in most early postoperative complications. At follow-up, symptom prevalence in children aged 2-7 with DREA ranged from $37.5 \%$ (heartburn) to $75 \%$ (cough). Further digestive and respiratory symptoms were present in $\geq 50 \%$. In children aged $8-18$, it ranged from $14.3 \%$ (vomiting) to $40.9 \%$ (cough), with other digestive and airway symptoms present in 19.0-27.3\%. Except for chest tightness(2-7 years), there were no significant differences in symptom prevalence between children with DREA and PA, nor between their generic or condition-specific HRQOL scores $(p>0.05)$. More children with DREA underwent esophageal dilatations (both age groups), gastrostomy feeding(2-7 years), and antireflux treatment (8-18 years), $p<0.05$. Days to hospital discharge after EA repair and a number of associated anomalies showed a strong negative correlation with HRQOL scores (2-7 years). Presence of cough, airway infection, swallowing difficulties and heartburn were associated with lower HRQOL scores (8-18 years), $\mathrm{p}<0.05$.

Conclusions Although children with DREA need more treatments, they are not a risk group for postoperative morbidity and impaired HRQOL compared with children with PA. However, those with a long initial hospital stay, several associated anomalies and digestive or respiratory symptoms risk worse HRQOL. This is important information for clinical practice, families and patient stakeholders.

\section{Background}

Esophageal atresia (EA) with or without a tracheoesophageal fistula (TEF) remains a rare condition with a prevalence of 2.4 in 10,000 live births [1]. Nevertheless, primary anastomosis of EA with distal TEF (PA) has become a standard procedure with over $90 \%$ survival rates [2]. In 10-15\% of cases, the reconstruction of EA is delayed, usually because the gap between the two esophageal ends is too long (long-gap EA, LGEA)[3, 4], or related to the neonate's degree of prematurity/birth weight [5-7]. Children who undergo delayed reconstruction of EA (DREA) represent a rare, complicated patient group with a high risk of future morbidity [3, 8-10].

Historically, LGEA is managed by inserting a gastrostomy for enteral feeding, allowing for spontaneous growth of the esophageal segments, then performing a delayed primary anastomosis (DPA) when the child is 3 to 4 months old[8]. The native esophagus can also be preserved following elongation techniques, like Foker's technique[11, 12] or Kimura's advancement [13]. Esophageal replacement (ER) may also be employed, using stomach, jejunum or colon and with the conduit of choice depending on the center where procedures are performed $[3,4,14]$. When neonates with EA are extremely premature and/or have very low to extremely low birth weight, primary or staged repairs are used[5, $7,15,16]$.

Children with LGEA as opposed to short-gap EA more commonly present with cardio-vascular malformations[17, 18], genetic syndromes, prematurity and low birth weight[17]. Moreover, they are at higher risk of developing postoperative esophageal strictures [19-21], gastroesophageal reflux disease (GERD)[22], feeding difficulties [23], including need for gastrostomy feeding[19, 24], malnutrition[25] and lung impairments[26, 27]. Likewise, premature children with EA are at higher risk of digestive and respiratory morbidity [9, 28], and requiring gastrostomy for long-term feeding[29].

Health-related quality of life (HRQOL) refers to the individual's perception of the impact of disease and treatment on physical, psychological, and social functioning and well-being[30]. Although HRQOL research in patients with EA has expanded in recent years, knowledge of HRQOL in children with DREA is scarce [31, 32]. One study demonstrated worse generic HRQOL in preschool children with isolated EA vs those with EA and distal TEF[33]. Other studies found similar HRQOL in EA children with jejunal interposition, gastric pull-up (GPU) [34] and thoracoscopic external traction technique[35] compared with healthy children. Another study showed higher HRQOL scores in EA children with DPA vs children with ER and healthy references[36]. Likewise, patients with EA and esophagocoloplasty [37] and gastric tube interposition have good HRQOL [38]. Additionally, studies confirm that prematurity [33, 39] is associated with impaired generic HRQOL in children with EA. Recently, age and condition-specific HRQOL questionnaires for children with EA were developed (the "EA-QOL questionnaires"). Following their validation[40-42], these have not yet been applied to children with DREA.

This study aimed to evaluate postoperative morbidity and age-specific generic as well as condition-specific HRQOL in Swedish children with DREA aged 2-7 and 8-18 years, including DPA and ER, comparing them with children who underwent PA. Within the DREA group, we also aimed to identify factors associated with lower HRQOL scores and assess parent-child agreement in rating the child's HRQOL.

\section{Methods}

Ethics

This study was approved by the Swedish Ethical Committee in 2019 (2019-04930) and 2020 (2020-04310).

\section{Setting}


In Sweden, an average of 32 infants are born annually with EA [43]. Historically, these children have been surgically treated at four tertiary pediatric surgical centers. The children are offered standardized follow-up care at a tertiary pediatric surgical center (ie a minimum amount of follow-up) according to a national follow-up program established in 2011 (Figure 1).

\section{Study participants}

Families of children with EA Gross type A (isolated EA), B (EA with proximal TEF), C (EA with distal TEF), Gross D (EA with proximal and distal TEF) were eligible for recruitment if the child was aged between 2-18 at the time of the study and they were fluent in written and spoken Swedish. Children aged $<8$ years and children with cognitive dysfunction, were represented by their parent-proxy reports only. Children aged $\geq 15$ years and legal guardians of children aged 2 18 years needed to give written informed consent to participate.

\section{Children with DREA}

Children were considered to have DREA when primary anastomosis was not achievable at the first operation either because it was too far between esophageal segments, or because of the degree of prematurity/birth weight, meaning that these children received a gastrostomy/jejunostomy for enteral feeding. Fortyfive children with DREA were identified through hospital records from the Karolinska University Hospital, Stockholm ( $n=15)$, the Uppsala University Hospital, Uppsala ( $n=14)$, Sahlgrenska University Hospital, Gothenburg $(n=13)$ and Skåne University Hospital, Lund ( $n=3)$. Their anatomical subtypes were Gross A $(n=19)$, Gross B ( $n=12)$ and Gross $C(n=14)$ and they underwent esophageal reconstruction with DPA ( $=18)$, gastric tube esophagoplasty preserving the distal esophageal segment $(n=12)$, partial GPU $(n=6), G P U(n=5)$ and colon interposition $(n=4)$. Of the families, two did not respond, one patient was deceased, one had moved abroad, one was excluded for social reasons, one for lack of skills in Swedish and five families declined to participate. Hence, the study included families of 34 children aged 2-18 with DREA (10 children aged 2-7 and 24 children aged 8-18.)

\section{Comparison group; children with Gross type $\mathrm{C}$ who underwent primary anastomosis}

The children with Gross type $\mathrm{C}$ who underwent PA were recruited from Sahlgrenska University Hospital, Gothenburg. They included 106 families (42 children aged 2-7; 64 children aged 8-18) who had participated in an earlier study of generic HRQOL [33] and/or the field test of the EA-QOL questionnaires[42] with $\geq 90 \%$ response rate, and served in a comparison group for children with DREA.

\section{Data collection}

Families of children with DREA received questionnaires with pre-stamped reply envelopes to increase response rates, and non-respondents received a maximum of three reminders. Data was collected from mid-January to March in 2020, then was paused due to the covid-19 pandemic. The last four replies were collected between February and April in 2021.

\section{Clinical data}

A researcher at each center reviewed medical records for birth characteristics, Gross EA-type, initial gap length measured in centimeters or vertebral bodies as available, associated anomalies, surgical interventions, reasons for delayed reconstruction, postoperative outcomes, time to esophageal reconstruction and to hospital discharge from the tertiary care. Data on the child's health, including presence of digestive and airway symptoms and medication intake the previous four weeks, were collected through a parent-reported questionnaire, which had also been used on children with PA[33, 42].

\section{Parent characteristics}

One parent of each child answered a survey asking for information about the participating parent, including parental age, marital status and educational level.

\section{Generic HRQOL}

Generic HRQOL was measured by PedsQL ${ }^{\mathrm{TM}} 4.0$ generic core scales (PedsQL ${ }^{\mathrm{TM}} 4.0$ ) which has been evaluated for use in healthy children and children with chronic conditions. The PedsQL 4.0 for children aged 2-4 comprises 21 items, while the versions for children aged 5-7, 8-12 and 13-18 years include 23 items. The aspects measured are physical (8 items), emotional ( 5 items), social ( 5 items), and school functioning ( 5 or 3 items). Questions are answered using a 4 week recall period, using a 5-point Likert scale $[44,45]$.

\section{Condition-specific HRQOL}

Condition-specific HRQOL was measured by the EA-QOL questionnaires, which were originally developed and validated in Sweden and Germany [40-42]. The version for children aged 2-7 consists of 17 items, the domains being eating ( 7 items), physical health \& treatment ( 6 items) and social isolation \& stress ( 4 items). The version for children aged 8-18 consists of 24 items, the domains being eating (8 items), social relationships (7 items), body perception (5 items) and health \& wellbeing (4 items). Questions were answered using a 4-week recall period, and a five-point Likert scale[40-42]. 


\section{Data analysis}

Data were analyzed using IBM SPSS Statistics for Windows (version 25.0, Armonk, NY, USA: IBM Corp) and SAS 9.4(SAS Institute Inc., Cary, NC, USA). The 5point Likert scale responses to the PedsQL ${ }^{\mathrm{TM}} 4.0[44,45]$ and the EA-QOL-questionnaires [42] were linearly transformed to a 0-100 scale, with higher levels denoting better HRQOL. We required $\geq 70 \%$ of item responses for scale score calculations. The children's HRQOL scores were analyzed in age groups $2-7$ (parent-report) and 8-18 (child-and-parent report respectively) in accordance with the instrument's design[42] and age for child self-report (8 years). For continuous variables, median and range were calculated and for categorial variables, frequencies and percentages. Tests of significance included nonparametric methods. Mann-Whitney U-test and the Kruskal-Wallis $\mathrm{H}$ test respectively, were used to determine if there were statistically significant differences between two or more groups, when the dependent variable was ordinal or continous (and when the subgroups had $\geq 5$ observations). Fisher's exact test was used to determine if there were associations between two categorical variables and Pearson Chi Square for more than two categorial variables. Spearman's rank correlation (Spearman's rho) was used for bivariate correlation analysis, with Spearman's rho considered weak (0-0.39), moderate (0.40-0.59), strong $(\geq 0.60)$. In children aged 8-18 with DREA, child-parent agreement in ratings of the child's HRQOL were calculated using intra-class correlation coefficient (ICC) with values considered moderate $(0.5-0.74)$, good $(0.75-0.9)$ and excellent $(>0.90)$. Significance level was considered at $p<0.05$.

\section{Results}

\section{Study participants}

Out of 34 families who accepted study participation, 30 families of children with DREA ( $n=8$ children aged 2-7 years; $n=22$ children aged 8-17 years) gave informed consent and returned the questionnaires. Median age at follow-up was similar in children with DREA to children with PA, both in the younger group (6 years vs 5 years, $p=0.24$ ) and in the older group (13 years vs 13 years, $p=0.68$ ). In this study, subgroup analysis of 2 to 7 -year-olds with DREA was not feasible due to low sample size.

\section{Congenital and parent-proxy characteristics}

Table 1 presents the congenital/neonatal characteristics of children with DREA and children with PA and characteristics of the parent acting as proxy. Additional file 1 shows this information in children aged 8-18 with DPA, ER and PA. 
Table 1

Congenital/neonatal and parent characteristics in children with delayed compared to primary reconstruction of esophageal atresia

Children aged 2-7 years: $n(\%)$

Children aged $8-18$ years: $n(\%)$

\begin{tabular}{|c|c|c|c|c|c|c|c|c|c|c|}
\hline Child characteristics & $n_{\text {tot }}$ & $\begin{array}{l}\text { Delayed } \\
\text { reconstruction }\end{array}$ & $\mathrm{n}_{\text {tot }}$ & $\begin{array}{l}\text { Primary } \\
\text { anastomosis }\end{array}$ & $\begin{array}{l}\mathrm{p}- \\
\text { value }\end{array}$ & $\mathrm{n}_{\text {tot }}$ & $\begin{array}{l}\text { Delayed } \\
\text { reconstruction }\end{array}$ & $\mathrm{n}_{\text {tot }}$ & $\begin{array}{l}\text { Primary } \\
\text { anastomosis }\end{array}$ & $\begin{array}{l}\mathrm{p}- \\
\text { value }\end{array}$ \\
\hline Child gender male & 8 & $5(62.5)$ & 42 & $27(64.3)$ & 1.0 & 22 & $10(45.5)$ & 64 & $34(53.1)$ & 0.62 \\
\hline $\begin{array}{l}\text { Gestational age weeks, } \\
\text { median (range) }\end{array}$ & 8 & $36(26-37)$ & 42 & $37(31-41)$ & 0.096 & 22 & $36(24-40)$ & 62 & $38(28-43)$ & 0.002 \\
\hline $\begin{array}{l}\text { Prematurely born }(<37 \\
\text { gestational weeks at } \\
\text { birth) }\end{array}$ & 8 & $5(62.5)$ & 42 & $19(45.2)$ & 0.46 & 22 & 13(59.1) & 62 & $17(27.4)$ & 0.010 \\
\hline $\begin{array}{l}\text { Birth weight grams, } \\
\text { median (range) }\end{array}$ & 8 & 2202(590- 2855) & 41 & $\begin{array}{l}2653(1614- \\
4260)\end{array}$ & 0.014 & 22 & 2312(525-3225) & 62 & $\begin{array}{l}2720(1070- \\
3390)\end{array}$ & 0.030 \\
\hline $\begin{array}{l}\text { Low birth weight }(<2500 \\
\text { grams at birth) }\end{array}$ & 8 & $7(87.5)$ & 41 & $21(51.2)$ & 0.12 & 22 & 13(59.1) & 62 & $18(29.5)$ & 0.020 \\
\hline $\begin{array}{l}\text { Gross type esophageal } \\
\text { atresia }\end{array}$ & 8 & $\begin{array}{l}A=3(37.5) \\
B=1(12.5) \\
C=4(50.0)\end{array}$ & 42 & $C=42(100)$ & - & 22 & $\begin{array}{l}A=9(40.9) \\
B=7(31.8) \\
C=6(27.3)\end{array}$ & 64 & $C=64(100)$ & - \\
\hline Associated anomalies $^{a}$ & 8 & $8(100)$ & 42 & $24(57.1)$ & 0.039 & 22 & $12(54.5)$ & 64 & $39(60.9)$ & 0.62 \\
\hline Cardiovascular & 8 & $5(62.5)$ & 42 & $9(21.4)$ & 0.030 & 22 & $5(22.7)$ & 64 & $22(34.4)$ & 0.43 \\
\hline Anorectal & 8 & $2(25.0)$ & 42 & $6(14.3)$ & 0.60 & 22 & $5(22.7)$ & 64 & $4(6.3)$ & 0.044 \\
\hline Urogenital & 8 & $4(50.0)$ & 42 & $4(9.5)$ & 0.016 & 22 & $7(31.8)$ & 64 & $8(12.5)$ & 0.053 \\
\hline VACTERL association ${ }^{b}$ & 8 & $3(37.5)$ & 42 & $6(14.3)$ & 0.14 & 22 & $3(13.6)$ & 64 & $11(17.2)$ & 1.0 \\
\hline Verified genetic disorder & 8 & $1(12.5)$ & 42 & $3(7.1)$ & 0.51 & 22 & $4(18.2)$ & 64 & $7(10.9)$ & 0.46 \\
\hline \multicolumn{11}{|l|}{$\begin{array}{l}\text { Reason for delayed } \\
\text { reconstruction }\end{array}$} \\
\hline $\begin{array}{l}\text { Initital gap length, median } \\
\text { cm (range) }\end{array}$ & 3 & $4(2-7)$ & & & & & & & & \\
\hline $\begin{array}{l}\text { Initital gap length, median } \\
\text { vertebral bodies (range) }\end{array}$ & 3 & $4(3.5-4)$ & & & & 19 & $4(2-6)^{c}$ & & & \\
\hline $\begin{array}{l}\text { Degree of prematurity, } \\
\text { median (range) }\end{array}$ & 2 & $29(26-31)$ & & & & 3 & $29(24-30)$ & & & \\
\hline \multicolumn{11}{|l|}{$\begin{array}{l}\text { Characteristics of parent } \\
\text { acting as proxy }\end{array}$} \\
\hline Parent respondent mother & 8 & $5(62.5)$ & 42 & $37(88.1)$ & 0.11 & 22 & 18(81.8) & 63 & $56(88.9)$ & 0.46 \\
\hline $\begin{array}{l}\text { Parental age median } \\
\text { (range }\end{array}$ & 8 & $40(35-47)$ & 42 & $37(26-46)$ & 0.09 & 22 & $46(33-58)$ & 63 & $44(33-69)$ & 0.26 \\
\hline Cohabitant partner & 7 & $6(85.7)$ & 42 & $38(90.5)$ & 0.55 & 22 & $21(91.5)$ & 63 & $50(79.4)$ & 0.10 \\
\hline $\begin{array}{l}\text { University/College } \\
\text { education }\end{array}$ & 8 & $4(50.0)$ & 42 & $23(54.8)$ & 1.0 & 22 & 13(59.1) & 63 & $31(49.2)$ & 0.47 \\
\hline
\end{tabular}

Children aged 2-7 with DREA, had a higher rate of associated anomalies than children with PA (100\% vs $57.1 \%$, $p=0.039)$, specifically cardiovascular (62.5\% vs $21.4 \%, p=0.030)$ and urogenital anomalies $(50.0 \%$ vs $9.5 \%, p=0.016)$.

Children aged 8-18 with DREA, were more likely to have been born prematurely ( $59.1 \%$ vs $27.4 \%, p=0.010)$, with low birth weight ( $59.1 \%$ vs $29.5 \%$, $p=0.020)$ and with anorectal malformations $(22.7 \%$ vs $6.3 \%$, $p=0.044)$ than children with PA. Specifically, preterm birth was more common in children with DPA than in children with PA and children with ER had more anorectal and urogenital malformations ( $<<0.05$, Additional file 1$)$.

No significant differences between children with DREA and PA were found regarding parent characteristics (Table 1). 
In the DREA group of children 2 to 7 years, two underwent DPA, three GPU and three gastric tube esophagoplasty preserving the distal esophagus. The reconstruction of EA took place at a median of 174 days (range 48-1221) and none had antireflux surgery at the time of esophageal reconstruction. In the DREA group aged 8-18 years, 12 children underwent DPA, five gastric tube esophagoplasty preserving the distal esophagus, three partial GPU and two colon interposition. Six children with ER had antireflux surgery at reconstruction. The delayed reconstruction took place at a median of 137 days (range 34-323), and there was no significant difference between DPA (median 113 days, range 34-323) and ER (median 164 days, range 36-314), p=0.29.

\section{Postoperative morbidity}

\section{Early postoperative complications}

Table 2 displays the postoperative course before discharge from a tertiary pediatric surgical ward for children with DREA vs children with PA. There were no significant differences between the groups for most of the complications assessed (Table 2).

Table 2

Postoperative course in children with delayed compared to primary reconstruction of esophageal atresia

\begin{tabular}{|c|c|c|c|c|c|c|c|c|c|c|}
\hline & \multicolumn{6}{|c|}{ Children aged $2-7$ years: $n(\%)$} & \multicolumn{4}{|c|}{ Children aged $8-18$ years: $n(\%)$} \\
\hline & $\mathrm{n}_{\text {tot }}$ & $\begin{array}{l}\text { Delayed } \\
\text { reconstruction }\end{array}$ & $\mathrm{n}_{\text {tot }}$ & $\begin{array}{l}\text { Primary } \\
\text { anastomosis, } \\
\text { Gross type C }\end{array}$ & p-value & $\mathrm{n}_{\text {tot }}$ & $\begin{array}{l}\text { Delayed } \\
\text { reconstruction }\end{array}$ & $\mathrm{n}_{\text {tot }}$ & $\begin{array}{l}\text { Primary } \\
\text { anastomosis, } \\
\text { Gross type C }\end{array}$ & p-value \\
\hline $\begin{array}{l}\text { Revisional surgery after repair of } \\
\text { EA eg due to anastomotic } \\
\text { leakage or recurrent fistula }\end{array}$ & 8 & $1(12.5)$ & 42 & $3(7.1)$ & 0.51 & 22 & $4(18.2)$ & 62 & $7(10.9)$ & 0.46 \\
\hline Anastomotic leakage & 8 & $1(12.5)$ & 42 & $2(4.8)$ & 0.41 & 22 & $8(36.4)$ & 62 & $8(12.9)$ & 0.026 \\
\hline $\begin{array}{l}\text { Sepsis verified through blood } \\
\text { culture }\end{array}$ & 8 & $4(50.0)$ & 42 & $8(19.0)$ & 0.082 & 22 & $6(27.3)$ & 62 & $6(9.7)$ & 0.071 \\
\hline Wound infection & 8 & $1(12.5)$ & 42 & $1(2.4)$ & 0.30 & 22 & $1(4.5)$ & 62 & $2(3.2)$ & 1.0 \\
\hline $\begin{array}{l}\text { Pneumothorax treated with } \\
\text { drainage }\end{array}$ & 8 & $3(37.5)$ & 42 & $2(4.8)$ & 0.024 & 22 & $3(13.6)$ & 62 & 11(17.7) & 0.75 \\
\hline $\begin{array}{l}\text { Esophageal dilatation before } \\
\text { hospital discharge }\end{array}$ & 5 & $2(20.0)$ & 42 & $1(2.4)$ & 0.20 & 20 & $2(10.0)$ & 60 & $8(13.3)$ & 1.0 \\
\hline $\begin{array}{l}\text { Days before discharge from } \\
\text { tertiary pediatric surgical care, } \\
\text { median (range) }\end{array}$ & 8 & $230(69-1235)$ & 42 & $31(4-231)$ & $<0.0001$ & 22 & $188(62-364)$ & 59 & $35(19-464)$ & $<0.0001$ \\
\hline
\end{tabular}

\section{Symptoms and treatment at follow-up}

Figure 2a-b compares the proportion of children with DREA with digestive or respiratory symptoms and their treatment at follow-up, with that of children with PA.

In children aged 2-7 with DREA, symptom prevalence ranged from 37.5\% (heartburn) to $75 \%$ (cough). There was a higher rate of chest tightness among children with DREA compared to those with PA ( $p=0.015)$. At follow-up, more children with DREA had gastrostomy feeding ( $p=0.005)$ and esophageal dilatations ( $p=0.015$ ), with a median of 4 (range $0-19$ ) compared to children with PA median of 0 (range $0-11$ ), $p<0.001$. They were rarely treated with antireflux surgery, but commonly with antireflux medication, inhaled steroids and/or bronchodilators.

In children aged 8-18 with DREA, symptom prevalence ranged from 14.3\%(vomiting) to $40.9 \%$ (cough). No signicant differences between children with DREA vs PA regarding symptom prevalence were found. At follow-up, gastrostomy feeding was rare in any group, but children with DREA had significantly more dilatations (median 6 , range $0-46$ ) than children with PA (median 0 , range $0-62$ ), $p<0.001$, as well as antireflux surgery and antireflux medication ( $p<0.05$ ). Moreover, within the DREA group, there were no significant differences in symptom prevalence between children with DPA and ER ( $p>0.05$ ). However, children with DPA were more commonly treated with esophageal dilatations $(p=0.012)$ than children with PA and more children with ER had antireflux surgery than children with PA $(p=0.008)$.

\section{HRQOL}

Additional file 2 presents descriptives for HRQOL scores in children with DREA and PA, complementary to Figures 3-6.

\section{Generic HRQOL}


Figure 3a-c compares the PedsQL 4.0 scores in children with DREA with children with PA. In children aged 2-7, the median scores for physical, social, school functioning and total generic HRQOL were numerically lower in children with DREA than with PA, but the differences were non-significant ( $p>0.05$ ). In children aged 8-18, there were no significant differences in generic HRQOL scores between children with DREA and PA ( $p>0.05)$ or as viewed in Figure $4 a-b$, between those with DPA, ER or PA ( $>0.05)$.

\section{Condition-specific HRQOL}

Figure 5a-c compares the EA-QOL scores in children with DREA with children with PA. In age group 2-7, all domain or total scores measured by the EA-QOL questionnaires demonstrated lower median scores in children with DREA than in children with PA, but differences were non-significant ( $p>0.05$ ). In age group 8-18, there were no significant differences in EA-QOL scores between children with DREA and PA ( $p>0.05)$, or as viewed in Figure 6a-b between those with DPA, ER or PA $(p>0.05)$.

\section{Factors associated with lower HRQOL scores}

Table 3 presents generic and condition-specific HRQOL in children aged 8-18 with DREA and with and without digestive and respiratory symptoms (in subgroups with $\geq 5$ observations). Swallowing difficulties, heartburn, cough or airway infections were significantly associated with lower generic and/or condition-specific HRQOL scores, $p<0.05$. Table 4 shows the correlation between clinical factors and HRQOL scores among children with DREA. The number of associated anomalies present in the child demonstrated a strong negative correlation with six HRQOL scales (physical functioning, emotional functioning, total PedsQL 4.0 scores, eating, physical health \& treatment and total EA-QOL scores) in children aged 2-7, p $<0.05$. Similarly, days to discharge from tertiary pediatric surgical ward showed a strong negative correlation with five HRQOL scales (physical functioning, social functioning, total PedsQL 4.0 scores, physical health \& treatment and total EA-QOL) in children aged 2-7, $\mathrm{p}<0.05$. 
Generic and condition-specific health-related quality of life in sympt

\begin{tabular}{|c|c|c|c|c|c|c|c|c|c|c|c|c|c|}
\hline & & \multicolumn{12}{|c|}{ Generic health-related quality of life } \\
\hline & & \multicolumn{5}{|c|}{ Physical functioning } & \multicolumn{5}{|c|}{ Emotional functioning } & \multicolumn{2}{|c|}{ Social } \\
\hline \multicolumn{2}{|c|}{$\begin{array}{l}\text { SYMPTOMS THE PAST } \\
\text { FOUR WEEKS }\end{array}$} & $\begin{array}{l}\text { n, } \\
\text { yes }\end{array}$ & Median(range) & $\begin{array}{l}\text { n, } \\
\text { no }\end{array}$ & Median(range) & $\mathrm{p}$-value & $\begin{array}{l}\text { n, } \\
\text { yes }\end{array}$ & Median(range) & $\begin{array}{l}\text { n, } \\
\text { no }\end{array}$ & Median(range) & $\begin{array}{l}\mathrm{p}- \\
\text { value }\end{array}$ & $\begin{array}{l}\mathrm{n}, \\
\text { yes }\end{array}$ & $\mathrm{M} \epsilon$ \\
\hline \multirow[t]{4}{*}{$\begin{array}{l}\text { CHILD- } \\
\text { REPORT }\end{array}$} & $\begin{array}{l}\text { Swallowing } \\
\text { difficulties }\end{array}$ & 5 & $\begin{array}{l}59.4(40.6- \\
100)\end{array}$ & 15 & $\begin{array}{l}93.8(65.6- \\
100)\end{array}$ & 0.21 & 5 & $\begin{array}{l}70.0(60.0- \\
100)\end{array}$ & 15 & $\begin{array}{l}90.0(65.0- \\
100)\end{array}$ & 0.37 & 5 & $\begin{array}{l}70 . \\
10 i\end{array}$ \\
\hline & Heartburn & 5 & $\begin{array}{l}87.5(40.6- \\
93.8)\end{array}$ & 15 & $100(56.3-100)$ & 0.054 & 5 & $\begin{array}{l}85.0(65.0- \\
100)\end{array}$ & 15 & $\begin{array}{l}95.0(60.0- \\
100)\end{array}$ & 0.35 & 5 & $\begin{array}{l}95 . \\
10 i\end{array}$ \\
\hline & Cough & 7 & $\begin{array}{l}87.5(40.6- \\
100)\end{array}$ & 13 & $100(65.6-100)$ & $0.046 *$ & 7 & $85.0(60.0-100)$ & 13 & $\begin{array}{l}95.0(65.0- \\
100)\end{array}$ & 0.29 & 7 & $\begin{array}{l}95 . \\
101\end{array}$ \\
\hline & $\begin{array}{l}\text { Airway } \\
\text { infections }\end{array}$ & 5 & $\begin{array}{l}84.4(40.6- \\
93.8)\end{array}$ & 15 & $100(59.4-100)$ & $0.025^{*}$ & 5 & $\begin{array}{l}70.0(60.0- \\
100)\end{array}$ & 15 & $\begin{array}{l}95.0(65.0- \\
100)\end{array}$ & 0.075 & 5 & $\begin{array}{l}70 . \\
101\end{array}$ \\
\hline \multirow[t]{6}{*}{$\begin{array}{l}\text { PARENT- } \\
\text { REPORT }\end{array}$} & $\begin{array}{l}\text { Swallowing } \\
\text { difficulties }\end{array}$ & 5 & $\begin{array}{l}56.3(56.3- \\
100)\end{array}$ & 17 & $\begin{array}{l}98.4(31.3- \\
100)\end{array}$ & 0.091 & 5 & $\begin{array}{l}80.0(55.0- \\
100)\end{array}$ & 17 & $\begin{array}{l}95.0(30.0- \\
100)\end{array}$ & 0.35 & 5 & $\begin{array}{l}67 . \\
10 i\end{array}$ \\
\hline & Heartburn & 6 & $\begin{array}{l}57.8(31.3- \\
87.5)\end{array}$ & 16 & $100(56.3-100)$ & $0.003 *$ & 6 & $\begin{array}{l}70.0(30.0- \\
100)\end{array}$ & 16 & $\begin{array}{l}95.0(55.0- \\
100)\end{array}$ & $0.041^{*}$ & 6 & $\begin{array}{l}67 . \\
10 i\end{array}$ \\
\hline & Cough & 8 & $\begin{array}{l}57.8(31.3- \\
87.5)\end{array}$ & 13 & $100(65.6-100)$ & $<0,001^{*}$ & 9 & $\begin{array}{l}80.0(30.0- \\
100)\end{array}$ & 13 & $\begin{array}{l}95.0(70.0- \\
100)\end{array}$ & $0.026^{*}$ & 8 & 67. \\
\hline & $\begin{array}{l}\text { Airway } \\
\text { infections }\end{array}$ & 6 & $\begin{array}{l}57.8(31.2- \\
100)\end{array}$ & 15 & $100(56.3-100)$ & $0.017 *$ & 6 & $\begin{array}{l}57.5(30.0- \\
100)\end{array}$ & 16 & $\begin{array}{l}95.0(70.0- \\
100)\end{array}$ & 0.096 & 6 & $\begin{array}{l}60 . \\
10 i\end{array}$ \\
\hline & & \multicolumn{12}{|c|}{ Condition-specific health-related quality of life } \\
\hline & & \multicolumn{5}{|c|}{ Eating } & \multicolumn{5}{|c|}{ Social relationships } & \multicolumn{2}{|c|}{ Body F } \\
\hline \multirow[t]{4}{*}{$\begin{array}{l}\text { CHILD- } \\
\text { REPORT }\end{array}$} & $\begin{array}{l}\text { Swallowing } \\
\text { difficulties }\end{array}$ & 5 & $\begin{array}{l}43.8(17.9- \\
59.4)\end{array}$ & 14 & $\begin{array}{l}81.3(53.1- \\
100)\end{array}$ & $0.002^{\star}$ & 5 & $\begin{array}{l}66.1(57.1- \\
85.7)\end{array}$ & 14 & $\begin{array}{l}91.1(42.9- \\
100)\end{array}$ & $0.048^{*}$ & 5 & $\begin{array}{l}90 . \\
95 .\end{array}$ \\
\hline & Heartburn & 5 & $\begin{array}{l}75.0(17.9- \\
84.4)\end{array}$ & 15 & $\begin{array}{l}78.1(43.8- \\
100)\end{array}$ & 0.29 & 5 & $\begin{array}{l}73.2(57.1- \\
89.3)\end{array}$ & 15 & $\begin{array}{l}89.3(32.1- \\
100)\end{array}$ & 0.27 & 5 & $\begin{array}{l}85 . \\
10 i\end{array}$ \\
\hline & Cough & 7 & $\begin{array}{l}59.4(17.9- \\
84.4)\end{array}$ & 13 & $\begin{array}{l}81.3(43.8- \\
100)\end{array}$ & 0.080 & 6 & $\begin{array}{l}73.2(57.1- \\
89.3)\end{array}$ & 13 & $\begin{array}{l}92.8 \text { (32.1- } \\
100)\end{array}$ & 0.13 & 7 & $\begin{array}{l}95 . \\
101\end{array}$ \\
\hline & $\begin{array}{l}\text { Airway } \\
\text { infections }\end{array}$ & 5 & $\begin{array}{l}59.4(17.9- \\
84.4)\end{array}$ & 15 & $\begin{array}{l}78.1(40.6- \\
100)\end{array}$ & 0.26 & 5 & $\begin{array}{l}66.1(42.9- \\
89.3)\end{array}$ & 15 & $\begin{array}{l}89.3(32.1- \\
100)\end{array}$ & 0.11 & 5 & $\begin{array}{l}95 . \\
101\end{array}$ \\
\hline \multirow[t]{4}{*}{$\begin{array}{l}\text { PARENT- } \\
\text { REPORT }\end{array}$} & $\begin{array}{l}\text { Swallowing } \\
\text { difficulties }\end{array}$ & 5 & $\begin{array}{l}50.0(30.0- \\
60.3)\end{array}$ & 16 & $\begin{array}{l}87.5(50.0- \\
100)\end{array}$ & $0.002^{*}$ & 5 & $\begin{array}{l}74.1(53.6- \\
89.3)\end{array}$ & 16 & $\begin{array}{l}96.4(42.9- \\
100)\end{array}$ & 0.15 & 5 & $\begin{array}{l}80 . \\
95 .\end{array}$ \\
\hline & Heartburn & 6 & $\begin{array}{l}51.6(30.0- \\
96.9)\end{array}$ & 16 & $\begin{array}{l}84.4(50.0- \\
100)\end{array}$ & $0.024^{*}$ & 5 & $\begin{array}{l}62.2(42.9- \\
71.4)\end{array}$ & 16 & $\begin{array}{l}94.6(46.4- \\
100)\end{array}$ & $0.020 *$ & 5 & $\begin{array}{l}65 . \\
80 .\end{array}$ \\
\hline & Cough & 9 & $\begin{array}{l}53.1(30.0- \\
96.9)\end{array}$ & 13 & $\begin{array}{l}90.6(50.0- \\
100)\end{array}$ & $0.004^{*}$ & 9 & $\begin{array}{l}71.4(42.9- \\
89.3)\end{array}$ & 13 & $100(46.4-100)$ & $0.039 *$ & 9 & $\begin{array}{l}72 . \\
95 .\end{array}$ \\
\hline & $\begin{array}{l}\text { Airway } \\
\text { infections }\end{array}$ & 6 & $\begin{array}{l}56.3(30.0- \\
96.9)\end{array}$ & 16 & $\begin{array}{l}84.4(40.6- \\
100)\end{array}$ & 0.10 & 5 & $\begin{array}{l}71.1(46.4- \\
89.3)\end{array}$ & 16 & $\begin{array}{l}94.6(42.9- \\
100)\end{array}$ & 0.12 & 5 & $\begin{array}{l}75 . \\
95 .\end{array}$ \\
\hline${ }^{*} \mathrm{p}$ & & & & & & & & & & & & & \\
\hline
\end{tabular}


Table 4

Correlation between HRQOL scores and clinical factors among children with delayed reconstruction of EA

\begin{tabular}{|c|c|c|c|c|c|c|}
\hline \multirow[b]{2}{*}{$\begin{array}{l}\text { GENERIC HEALTH-RELATED } \\
\text { QUALITY OF LIFE }\end{array}$} & \multicolumn{6}{|c|}{ Spearman's rho } \\
\hline & $\begin{array}{l}\text { gestational } \\
\text { weeks at } \\
\text { birth }\end{array}$ & $\begin{array}{l}\text { birth } \\
\text { weight } \\
\text { grams }\end{array}$ & $\begin{array}{l}\text { Number of } \\
\text { associated } \\
\text { anomalies }^{a}\end{array}$ & $\begin{array}{l}\text { days to } \\
\text { reconstruction }\end{array}$ & $\begin{array}{l}\text { days to discharge from } \\
\text { tertiary pediatric surgical } \\
\text { ward }\end{array}$ & $\begin{array}{l}\text { number of } \\
\text { esophageal } \\
\text { dilatations }\end{array}$ \\
\hline \multicolumn{7}{|c|}{$\begin{array}{l}\text { Children aged } 2-7 \text { years (parent- } \\
\text { reports, } n=8 \text { ) }\end{array}$} \\
\hline Physical functioning & -0.38 & -0.61 & $-0.83^{*}$ & -0.68 & $-0.76 *$ & 0.074 \\
\hline Emotional functioning & -0.61 & $-0.74^{*}$ & $-0.80 *$ & -0.62 & -0.68 & 0.11 \\
\hline Social functioning & -0.15 & -0.27 & -0.72 & -0.52 & $-0.78 *$ & 0.12 \\
\hline School functioning & -0.15 & -0.31 & -0.72 & -0.46 & -0.71 & 0.055 \\
\hline Total PedsQL 4.0 scores & -0.34 & -0.60 & $-0.80 *$ & -0.62 & $-0.79 *$ & -0.024 \\
\hline \multicolumn{7}{|c|}{$\begin{array}{l}\text { Children aged } 8-18 \text { years (child- } \\
\text { reports, } n=20 / \text { parent-reports, } n=22 \text { ) }\end{array}$} \\
\hline Physical functioning & $0.04 /-0.06$ & $0.13 /-0.03$ & $-0.40 /-0.36$ & $0.18 / 0.23$ & $-0.043 /-0.007$ & $0.042 / 0.37$ \\
\hline Emotional functioning & $-0.12 / 0.15$ & $0.20 / 0.29$ & $-0.052 /-0.25$ & $0.14 /-0.46$ & $0.063 /-0.16$ & $0.053 / 0.33$ \\
\hline Social functioning & $0.04 / 0.023$ & $0.23 / 0.28$ & $-0.15 /-0.05$ & $0.21 / 0.04$ & $-0.02 / 0.041$ & $-0.12 / 0.14$ \\
\hline School functioning & $-0.04 /-0.003$ & $0.008 / 0.05$ & $-0.25 /-0.29$ & $0.051 /-0.08$ & $-0.15 /-0.11$ & $0.11 / 0.27$ \\
\hline Total PedsQL 4.0 scores & $-0.07 / 0.04$ & $0.03 / 0.11$ & $-0.37 /-0.37$ & $0.14 / 0.06$ & $-0.14 /-0.12$ & $0.082 / 0.26$ \\
\hline \multicolumn{7}{|l|}{$\begin{array}{l}\text { CONDITION-SPECIFIC HEALTH- } \\
\text { RELATED QUALITY OF LIFE }\end{array}$} \\
\hline \multicolumn{7}{|c|}{$\begin{array}{l}\text { Children aged } 2-7 \text { years (parent- } \\
\text { reports, } n=8 \text { ) }\end{array}$} \\
\hline Eating & -0.67 & -0.72 & $-0.81 *$ & -0.64 & -0.71 & -0.074 \\
\hline Physical health \& treatment & -0.39 & -0.62 & $-0.80 *$ & -0.68 & $-0.74^{*}$ & 0.024 \\
\hline Social isolation \& stress & 0.38 & 0.10 & -0.40 & -0.30 & -0.65 & -0.077 \\
\hline Total EA-QOL & -0.34 & -0.54 & $-0.78 *$ & -0.57 & $-0.83^{*}$ & -0.073 \\
\hline \multicolumn{7}{|c|}{$\begin{array}{l}\text { Children aged } 8-18 \text { years (child- } \\
\text { reports, } n=20 / \text { parent-reports, } n=22 \text { ) }\end{array}$} \\
\hline Eating & $0.10 / 0.10$ & $0.23 / 0.17$ & $-0.10 /-0.12$ & $-0.018 /-0.12$ & $-0.17 /-0.24$ & $-0.001 / 0.10$ \\
\hline Social relationships & $0.10 / 0.13$ & $0.12 / 0.028$ & $-0.02 / 0.28$ & $0.19 / 0.11$ & $-0.10 /-0.04$ & $0 / 0.02$ \\
\hline Body perception & $0.21 / 0.15$ & $0.25 / 0.037$ & $-0.1 / 0.24$ & $0.07 / 0.35$ & $-0.18 / 0.08$ & $-0.096 / 0.03$ \\
\hline Health \& well-being & $0.10 /-0.01$ & $0.02 /-0.03$ & $-0.53 * /-0.35$ & $-0.08 /-0.08$ & $-0.37 /-0.28$ & $0.21 / 0.37$ \\
\hline Total EA-QOL & $0.13 / 0.07$ & $0.24 / 0.09$ & $-0.14 / 0.003$ & $0.13 / 0.04$ & $-0.12 /-0.14$ & $0.07 / 0.17$ \\
\hline \multicolumn{7}{|c|}{ HRQOL= HEALTH-RELATED QUALITY OF LIFE, EA= Esophageal atresia } \\
\hline \multicolumn{7}{|c|}{ Spearman's rho considered weak ( $0-0.39)$, moderate $(0.40-0.59)$, strong $(\geq 0.60)$} \\
\hline
\end{tabular}

\section{Child-parent agreement regarding the child's HRQOL}

Table 5 presents the parent-child agreement in ratings of the child's generic and condition-specific HRQOL in children aged 8-18 with DREA, with the ICCs indicating moderate to good parent-child agreement. 
Table 5

Parent-child agreement in ratings of the child's generic and condition-specific HRQOL in children aged 8-18 years with delayed reconstruction of esophageal atresia

\begin{tabular}{|ll|}
\hline \multicolumn{2}{|l|}{ Intraclass correlation coefficients average measures } \\
\hline Generic health-related quality of life (PedsQL 4.0) & \\
\hline Physical functioning & 0.80 \\
\hline Emotional functioning & 0.68 \\
\hline Social functioning & 0.61 \\
\hline School functioning & 0.87 \\
\hline Total PedsQL 4.0 scores & 0.76 \\
\hline Conditions-specific health-related quality of life (EA-QOL) & \\
\hline Eating & 0.88 \\
\hline Social relationships & 0.81 \\
\hline Body perception & 0.74 \\
\hline Health \& well-being & 0.88 \\
\hline Total EA-QOL scores & 0.84 \\
\hline Intraclass correlation coefficients considered moderate $(0.5-0.74)$ good $(0.75-0.9)$ and excellent $(>0.90)$ \\
\hline
\end{tabular}

\section{Discussion}

This is the first study to report postoperative morbidity and generic as well as condition-specific HRQOL of life in children with DREA, using a national wide recruitment and comparing outcomes to children with PA of the same age group and gender distribution. Overall, we found that children with DREA do not present with more long-term digestive and respiratory morbidity or impaired HRQOL than children with PA.

Among our participants, the reconstruction was delayed mostly because of LGEA, which commonly refers to a gap length of $\geq 2-3 \mathrm{~cm}$ or $\geq 3$ vertebral bodies $[3,8]$ and although it is debated, LGEA can entail Gross type A, B and C [4], which is confirmed in our study. In line with previous literature on LGEA [17, 18], DREA was related to a higher frequency of associated anomalies in children aged 2-7 as well as to prematurity and low birth weight in children aged 8-18. However, in contrast $[17,18]$, genetic disorders were similarly present in children with DREA and PA. In our study sample, $25 \%$ of children aged $2-7$ and $54 \%$ of those aged 8-18 had DPA, which has been advocated as the best choice in LGEA [46, 47]. Evidence for one conduit being superior to another is weak [3, 4]. Altogether, gastric tube was most used, but the Swedish sample showed variety regarding ER. Currently, GPU is favored by several institutions, probably due to its technical safety[8] and has been introduced on Swedish children aged 2-7.

We observed that early postoperative complications were generally more common in children with DREA, but differences with children with PA were mostly non-significant. In terms of late morbidity, we found that cough was the most reported symptom in children with DREA, possibly due to the relationship with tracheomalacia, GERD, esophageal strictures, airway infections and asthma [48]. In children aged 2-7 with DREA, the least reported symptom was heartburn and antireflux medication was commonly used. Antireflux surgery however, was rarely employed in children aged 2-7, which is in line with recent studies suggesting restrictiveness [49]. In children aged 8-18 with DREA, the least reported symptom was vomiting, but the use of antireflux treatment may serve as explanation. A majority of children with DREA were treated with dilatations at follow-up. In age group 8-18, esophageal dilatations were most common after DPA, which is in agreement with findings by Stadil et al[50]. In follow-ups of children with DREA several children aged 2-7 were still dependent on gastrostomy feeding, unlike children aged 8-18.

Interestingly, when comparing the presence of respiratory or digestive symptoms in children with DREA and PA, there were very few significant differences. In children aged 2-7, these symptoms and medical treatments were common in both groups[22, 28, 51], which may explain these findings. Though, chest tightness was more frequent in children with DREA. Perhaps the use of GPU, which has previously been associated with chest tightness can add to the explanation[52]. In children aged 8-18, no significant differences in symptom prevalence between children with DREA and PA were seen, despite a high frequency of GER(D) and strictures being reported in patients with DPA, gastric tube and partial GPU [52, 53]. Since 2011, children with EA in Sweden are offered a standardized follow-up according to a pediatric surgical programme, and more care when needed. Children with PA were recruited from a center which has applied a standardized follow-up programme since the late 1990s. Nevertheless, more children aged 8-18 with DREA than with PA were treated for digestive morbidity and $58 \%$ used inhaled steroids and/or bronchodilators. This could imply that children with DREA, a group where complications are expected, have received more intense follow-up/treatments. In turn, this may explain their comparable symptom prevalence to children with PA.

Our study findings comparing HRQOL in children with DREA and PA agree with most previous studies showing similar levels between patients with LGEA/complicated EA and those with PA or with healthy references [32, 34-36]. Although these studies differ in design, recruitment strategies, HRQOL assessments and subgroups of children with LGEA/complex EA, they all focus on a complicated group of patients with EA. As previously discussed[34, 36, 54-56], the HRQOL results may be explained by the congenital nature of EA, where disease-related challenges become a part of the children's identity [57] and 
adaptation $[58,59]$. There are only two studies of coping used by children with EA [58,59], and these demonstrate that already as toddlers they use coping strategies in several disease-specific contexts. Their use of coping strategies is related to the severity of EA and can impact the children's HRQOL both positively and negatively[58]. Hence, there should be more research into coping as a possible factor influencing HRQOL in children with EA.

In children aged 8-18 with DREA, the presence of digestive or respiratory symptoms were associated with worse generic and/or condition-specific HRQOL, as in children with EA in general [42,54,60]. Like Gallo et al [34], we could not confirm a relationship between esophageal dilatations and HRQOL. Although esophageal dilatations may reflect disease severity, treatment aims to relieve troublesome symptoms [61], which could be a reason for our findings. Moreover, prematurity and low birth weight were not associated with impaired HRQOL in children with DREA. This differs to findings in studies including complicated/complex and mild cases of EA[33,39], where these variables could be interlinked with LGEA and associated anomalies. To the authors' knowledge, we are the first to show that an initial long hospital stay on a tertiary pediatric surgical ward, and a number of associated anomalies, acting as possible markers of disease severity negatively influenced HRQOL in children aged 2-7 with DREA. Moreover, in the DREA group, child/parent agreement as to the child's HRQOL was acceptable, in line with studies including children with mild and complicated EA[62].

\section{Limitations}

As in other studies[3, 4, 14], surgical treatments of DREA in Sweden vary according to institution and surgeon. Elongation techniques such as Foker [11, 12] or Kimura [13], jejunal interposition or thoracoscopic repair have not yet been introduced, which may differ to other countries. Despite nation-wide recruitment, the study sample is small, but larger than several HRQOL studies, including $\leq 10$ children in subgroups of complex EA[34-36]. Study sample inclusion was $30 / 45(67 \%)$, the overall response rate $30 / 34(88 \%)$ and respondents and non-respondents had similar Gross type and surgical procedures. Still, the number of non-participants weakens the generalizability of findings. The group of children is heterogenous in relation to indications for DREA, anatomical subtype, gap measurement, prematurity and associated anomalies. However, if we had applied more exclusion criteria to increase sample homogeneity, the study size a national-wide Swedish study would have been limited. The study did not use a control group of healthy children. Although we paused data collection until the implications of the covid-19 pandemic were better understood, the situation could hypothetically impact the condition-specific HRQOL situations the children encounter, as well as generic HRQOL[40, 63].

\section{Conclusions}

In a nation-wide Swedish setting, children with DREA do not overall present with more long-term postoperative morbidity or lower generic and conditionspecific HRQOL than children with PA of the same age group and gender distribution. This supports an understanding that children with DREA are not necessarily a risk group for impaired HRQOL compared with children with PA. However, in children with DREA, risk factors for impaired HRQOL may be an initial long hospital stay, several associated anomalies and persistent airway and digestive symptoms. Moreover, parents can probably be a reliable source of information, complementary to self-reporting in ages 8-18. This is important and encouraging information for clinical practice, parents, children and patient stakeholders. Nevertheless, there is a need for an international multicenter study focusing on HRQOL, coping/adaption and health care experiences in children with LGEA/complex EA and PA.

\section{Abbreviations}

DREA, Delayed reconstruction of esophageal atresia

DPA, Delayed primary anastomosis

EA, Esophageal atresia

EA-QOL, Esophageal-Atresia-quality-of life

ER, Esophageal replacement

GERD, Gastroesophageal reflux disease

GPU, Gastric pull-up

HRQOL, Health-related quality of life

LGEA, Long-gap esophageal atresia

PA, Primary anastomosis

PedsQL $4.0^{T M}$, Pediatric quality of life inventory

TEF, Tracheo-esophageal fistula

\section{Declarations}

\section{Ethics approval and consent to participate}

Page 11/18 


\section{Consent for publication}

Not applicable.

\section{Availability of data and material}

The datasets analyzed during the current study are available in the manuscript or in its additonal files. Further information is not available in public due to lack of ethical approval.

\section{Competing interests}

The authors declare no competing interests.

\section{Funding}

This study was funded by ALF Grants from Region of Västra Götaland (ALFGBG-942815; ALFGBG-964986), the "Petter Silfverskiölds minnesfond" foundation (2021-292) and HKH Kronprinsessan Lovisas förening för barnasjukvård/Stiftelsen Axel Tielmans Minnesfond (2021-00626). All authors have identified they have no financial relationships to disclose relevant to this study and its results.

\section{Authors' contributions}

MDB is the principal investigator of the study. MDB, SÖA, EÖ, JFS, AMK, LJ, KA, VG, PS, AMT, HEL developed the study design. MDB, SÖA, EÖ, AMT, JFS, AMK, $P S, H E L$ acquired the data. SÖA coordinated the national data acquisition with supervision from MDB, who also analyzed the data with a senior biostatistician. MDB, SÖA, EÖ, JFS. AMK, LJ, KA, VG, PS, AMT, EO, HEL interpreted the data. MDB wrote the draft of the manuscript which was reviewed critically for important intellectual content first by HEL, then by SÖA, EÖ, JFS, AMK, LJ, KA, VG, PS, AMT, EO. Before submission, all authors have reviewed the revised draft critically, approved the final manuscript to be published, and agreed to be accountable for all aspects of the work. All authors read and approved the final manuscript.

\section{Acknowledgements}

We would like to thank all participating families for sharing their perceptions and experiences and Statistiska Konsultgruppen, Gothenburg, Sweden for professional statistical support.

\section{References}

1. Pedersen RN, Calzolari E, Husby S, Garne E. Oesophageal atresia: prevalence, prenatal diagnosis and associated anomalies in 23 European regions. Arch Dis Child. 2012;97(3):227-32.

2. Zimmer J, Eaton S, Murchison LE, De Coppi P, Ure BM, Dingemann C. State of Play: Eight Decades of Surgery for Esophageal Atresia. Eur J Pediatr Surg. 2019;29(1):39-48.

3. Dingemann C, Eaton S, Aksnes G, Bagolan P, Cross KM, De Coppi P, et al. ERNICA Consensus Conference on the Management of Patients with Long-Gap Esophageal Atresia: Perioperative, Surgical, and Long-Term Management. Eur J Pediatr Surg 2021;31(3):214-25.

4. Baird R, Lal DR, Ricca RL, Diefenbach KA, Downard CD, Shelton J, et al. Management of long gap esophageal atresia: A systematic review and evidencebased guidelines from the APSA Outcomes and Evidence Based Practice Committee. J Pediatr Surg. 2019;54(4):675-87.

5. Zani A, Wolinska J, Cobellis G, Chiu PP, Pierro A. Outcome of esophageal atresia/tracheoesophageal fistula in extremely low birth weight neonates ( $<1000$ grams). Pediatr Surg Int. 2016;32(1):83-8.

6. Hannon EJ, Billington J, Kiely EM, Pierro A, Spitz L, Cross K, et al. Oesophageal atresia is correctable and survivable in infants less than 1 kg. Pediatr Surg Int. 2016;32(6):571-6.

7. Petrosyan M, Estrada J, Hunter C, Woo R, Stein J, Ford HR, et al. Esophageal atresia/tracheoesophageal fistula in very low-birth-weight neonates: improved outcomes with staged repair. Pediatr Surg. 2009;44(12):2278-81.

8. Shieh HF, Jennings RW. Long-gap esophageal atresia. Semin Pediatr Surg. 2017;26(2):72-7.

9. Rayyan M, Embrechts M, Van Veer H, Aerts R, Hoffman I, Proesmans M, et al. Neonatal factors predictive for respiratory and gastro-intestinal morbidity after esophageal atresia repair. Pediatr Neonatol. 2019;60(3):261-9.

10. Spitz L. Oesophageal atresia. Orphanet J Rare Dis. 2007;2:24.

11. Foker JE, Linden BC, Boyle EM Jr, Marquardt C. Development of a true primary repair for the full spectrum of esophageal atresia. Ann Surg. 1997;226(4):533-41.

Page $12 / 18$ 
12. Foker JE, Kendall TC, Catton K, Khan KM. A flexible approach to achieve a true primary repair for all infants with esophageal atresia. Semin Pediatr Surg. 2005;14(1):8-15.

13. Kimura K, Soper RT. Multistaged extrathoracic esophageal elongation for long gap esophageal atresia. J Pediatr Surg. 1994;29(4):566-8.

14. Zani A, Eaton S, Hoellwarth ME, Puri P, Tovar J, Fasching G, et al. International survey on the management of esophageal atresia. Eur J Pediatr Surg. 2014;24(1):3-8.

15. Schmidt A, Obermayr F, Lieber J, Gille C, Fideler F, Fuchs J. Outcome of primary repair in extremely and very low-birth-weight infants with esophageal atresia/distal tracheoesophageal fistula. J Pediatr Surg. 2017;52(10):1567-70.

16. Seitz G, Warmann SW, Schaefer J, Poets CF, Fuchs J. Primary repair of esophageal atresia in extremely low birth weight infants: a single-center experience and review of the literature. Biol Neonate. 2006;90(4):247-51.

17. Bairdain S, Zurakowski D, Vargas SO, Stenquist N, McDonald M, Towne MC, et al. Long-Gap Esophageal Atresia Is a Unique Entity within the Esophageal Atresia Defect Spectrum. Neonatology. 2017;111(2):140-4.

18. Aslanabadi S, Ghabili K, Rouzrokh M, Hosseini MB, Jamshidi M, Adl FH, et al. Associated congenital anomalies between neonates with short-gap and long-gap esophageal atresia: a comparative study. I J Gen Med. 2011;4:487-91.

19. Shah R, Varjavandi V, Krishnan U. Predictive factors for complications in children with esophageal atresia and tracheoesophageal fistula. Dis Esophagus. 2015;28(3):216-23.

20. Vergouwe FWT, Vlot J, Spaander HIJ, van Rosmalen MCW, Oomen J. MWN, et al. Risk factors for refractory anastomotic strictures after oesophageal atresia repair: a multicentre study. Arch Dis Child. 2019;104(2):152-7.

21. Lu YH, Yen TA, Chen CY, Tsao PN, Lin WH, Hsu WM, et al. Risk factors for digestive morbidities after esophageal atresia repair. Eur J Pediatr. 2021;180(1):187-94.

22. Svoboda E, Fruithof J, Widenmann-Grolig A, Slater G, Armand F, Warner B, et al. A patient led, international study of long term outcomes of esophageal atresia: EAT 1. J Pediatr Surg. 2018;53(4):610-5.

23. Baird R, Levesque D, Birnbaum R, Ramsay M. A pilot investigation of feeding problems in children with esophageal atresia. Dis Esophagus. 2015;28(3):224-8.

24. Castilloux J, Noble AJ, Faure C. Risk factors for short- and long-term morbidity in children with esophageal atresia. J Pediatr. 2010;156(5):755-60.

25. Pelizzo G, Destro F, Selvaggio GGO, Maestri L, Roveri M, Bosetti A, et al. Esophageal Atresia: Nutritional Status and Energy Metabolism to Maximize Growth Outcome. Children (Basel, Switzerland) 2020;7(11).

26. Jönsson L, Friberg LG, Gatzinsky V, Kötz K, Sillén U, Abrahamsson K. Treatment and Follow-Up of Patients with Long-Gap Esophageal Atresia: 15 Years' of Experience from the Western Region of Sweden. Eur J Pediatr Surg. 2016;26(2):150-9.

27. Gallo G, Vrijlandt E, Arets HGM, Koppelman GH, Van der Zee DC, Hulscher JBF, et al. Respiratory function after esophageal replacement in children. J Pediatr Surg. 2017;52(11):1736-41.

28. Ax S, Abrahamsson K, Gatzinsky V, Jönsson L, Dellenmark-Blom M. Parent-Reported Feeding Difficulties among Children Born with Esophageal Atresia: Prevalence and Early Risk Factors. Eur J Pediatr Surg. 2021;31(1):69-75.

29. Lazow SP, Ben-Ishay O, Aribindi VK, Staffa SJ, Pluchinotta FR, Schecter SC, et al. Predictors of index admission mortality and morbidity in contemporary esophageal atresia patients. J Pediatr Surg. 2020;55(11):2322-8.

30. Haverman L, Limperg PF, Young NL, Grootenhuis MA, Klaassen RJ. Paediatric health-related quality of life: what is it and why should we measure it? Arch Dis Child. 2017;102(5):393-400.

31. Dellenmark-Blom M, Quitmann J, Dingemann C. Health-Related Quality of Life in Patients after Repair of Esophageal Atresia: A Review of Current Literature. Eur J Pediatr Surg. 2020;30(3):239-50.

32. Tan Tanny SP, Comella A, Hutson JM, Omari TI, Teague WJ, King SK. Quality of life assessment in esophageal atresia patients: a systematic review focusing on long-gap esophageal atresia. J Pediatr Surg. 2019;54(12):2473-8.

33. Flieder S, Dellenmark-Blom M, Witt S, Dingemann C, Quitmann JH, Jönsson L, et al. Generic Health-Related Quality of Life after Repair of Esophageal Atresia and Its Determinants within a German-Swedish Cohort. Eur J Pediatr Surg. 2019;29(1):75-84.

34. Gallo G, van Tuyll van Serooskerken ES, Tytgat S, van der Zee DC, Keyzer-Dekker CMG, Zwaveling S, et al. Quality of life after esophageal replacement in children. J Pediatr Surg. 2021;56(2):239-44.

35. van Tuyll van Serooskerken ES, Lindeboom MYA, Verweij JW, van der Zee DC, Tytgat S. Childhood outcome after correction of long-gap esophageal atresia by thoracoscopic external traction technique. J Pediatr Surg. 2021;56(10):1745-51.

36. Dingemann C, Meyer A, Kircher G, Boemers TM, Vaske B, Till H, et al. Long-term health-related quality of life after complex and/or complicated esophageal atresia in adults and children registered in a German patient support group. J Pediatr Surg. 2014;49(4):631-8.

37. Tannuri ACA, Angelo SS, Takyi P, da Silva AR, Tannuri U. Esophageal substitution or esophageal elongation procedures in patients with complicated esophageal atresia? Results of a comparative study. J Pediatr Surg. 2021;56(5):933-7.

38. Youn JK, Park T, Kim SH, Han JW, Jang HJ, Oh C, et al. Prospective evaluation of clinical outcomes and quality of life after gastric tube interposition as esophageal reconstruction in children. Medicine. 2018;97(52):e13801.

39. Rozensztrauch A, Śmigiel R, Patkowski D. Congenital Esophageal Atresia-Surgical Treatment Results in the Context of Quality of Life. Eur J Pediatr Surg. 2019;29(3):266-70. 
40. Dellenmark-Blom M, Chaplin JE, Gatzinsky V, Jönsson L, Wigert H, Apell J, et al. Health-related quality of life experiences among children and adolescents born with esophageal atresia: Development of a condition-specific questionnaire for pediatric patients. J Pediatr Surg. 2016;51(4):563-9.

41. Dellenmark-Blom M, Abrahamsson K, Quitmann JH, Sommer R, Witt S, Dingemann J, et al. Development and pilot-testing of a condition-specific instrument to assess the quality-of-life in children and adolescents born with esophageal atresia. Dis Esophagus. 2017;30(7):1-9.

42. Dellenmark-Blom M, Dingemann J, Witt S, Quitmann JH, Jönsson L, Gatzinsky V, et al. The Esophageal-Atresia-Quality-of-life Questionnaires: Feasibility, Validity and Reliability in Sweden and Germany. J Pediatr Gastroenterol Nutr. 2018;67(4):469-77.

43. The National Board of Health and Welfare. Birth defects 2016. Annual reports from the Swedish Birth Defects Registry. Stockholm, Sweden; 2018.

44. Varni JW, Seid M, Kurtin PS. PedsQL 4.0: reliability and validity of the Pediatric Quality of Life Inventory version 4.0 generic core scales in healthy and patient populations. Med Care. 2001;39(8):800-12.

45. Petersen S, Hägglöf B, Stenlund H, Bergström E. Psychometric properties of the Swedish PedsQL, Pediatric Quality of Life Inventory 4.0 generic core scales. Acta Paediatr. 2009;98(9):1504-12.

46. van der Zee DC, Bagolan P, Faure C, Gottrand F, Jennings R, Laberge JM, et al. Position Paper of INoEA Working Group on Long-Gap Esophageal Atresia: For Better Care. Front Pediatr. 2017;5:63.

47. Reinberg O. Esophageal replacements in children. Ann NY Acad Sci. 2016;1381(1):104-12.

48. Koumbourlis AC, Belessis Y, Cataletto M, Cutrera R, DeBoer E, Kazachkov M, et al. Care recommendations for the respiratory complications of esophageal atresia-tracheoesophageal fistula. Pediatr Pulmonol. 2020;55(10):2713-29.

49. Jönsson L, Dellenmark-Blom M, Enoksson O, Friberg LG, Gatzinsky V, Sandin A, et al. Long-Term Effectiveness of Antireflux Surgery in Esophageal Atresia Patients. Eur J Pediatr Surg. 2019;29(6):521-7.

50. Stadil T, Koivusalo A, Svensson JF, Jönsson L, Lilja HE, Thorup JM, et al. Surgical treatment and major complications Within the first year of life in newborns with long-gap esophageal atresia gross type A and B - a systematic review. J Pediatr Surg. 2019;54(11):2242-9.

51. Schneider A, Blanc S, Bonnard A, Khen-Dunlop N, Auber F, Breton A, et al. Results from the French National Esophageal Atresia register: one-year outcome. Orphanet J Rare Dis. 2014;9:206.

52. Liu J, Yang Y, Zheng C, Dong R, Zheng S. Surgical outcomes of different approaches to esophageal replacement in long-gap esophageal atresia: A systematic review. Medicine. 2017;96(21):e6942.

53. Reismann M, Granholm T, Ehrén H. Partial gastric pull-up in the treatment of patients with long-gap esophageal atresia. World journal of pediatrics: WJP. 2015;11(3):267-71.

54. Legrand C, Michaud L, Salleron J, Neut D, Sfeir R, Thumerelle C, et al. Long-term outcome of children with oesophageal atresia type III. Arch Dis Child. 2012;97(9):808-11.

55. Deurloo JA, Klinkenberg EC, Ekkelkamp S, Heij HA, Aronson DC. Adults with corrected oesophageal atresia: is oesophageal function associated with complaints and/or quality of life? Pediatr Surg Int. 2008;24(5):537-41.

56. Ure BM, Slany E, Eypasch EP, Weiler K, Troidl H, Holschneider AM. Quality of life more than 20 years after repair of esophageal atresia. J Pediatr Surg. 1998;33(3):511-5.

57. Bogart KR. The Role of Disability Self-Concept in Adaptation to Congenital or Acquired Disability. Rehabil Psychol. 2014;59(1):107-15.

58. Dellenmark-Blom M, Chaplin JE, Jönsson L, Gatzinsky V, Quitmann JH, Abrahamsson K. Coping strategies used by children and adolescents born with esophageal atresia - a focus group study obtaining the child and parent perspective. Child Care Health Dev. 2016;42(5):759-67.

59. Dellenmark-Blom M, Chaplin JE, Quitmann JH, Jönsson L, Gatzinsky V, Dingemann J, et al. The prevalence and role of coping strategies in the nutritional intake of children born with esophageal atresia: a condition-specific approach. Dis Esophagus 2019;32(7).

60. Dellenmark-Blom M, Quitmann J, Dingemann J, Witt S, Ure BM, Bullinger M, et al. Clinical Factors Affecting Condition-Specific Quality-of-Life Domains in Pediatric Patients after Repair of Esophageal Atresia: The Swedish-German EA-QOL Study. Eur J Pediatr Surg. 2020;30(1):96-103.

61. Tambucci R, Angelino G, De Angelis P, Torroni F, Caldaro T, Balassone V, et al. Anastomotic Strictures after Esophageal Atresia Repair: Incidence, Investigations, and Management, Including Treatment of Refractory and Recurrent Strictures. Front Pediatr. 2017;5:120.

62. Witt S, Dellenmark-Blom M, Kuckuck S, Dingemann J, Abrahamsson K, Dingemann C, et al. Parent-child-agreement on health-related quality of life and its determinants in patients born with Esophageal Atresia: a Swedish-German cross-sectional study. Orphanet J Rare Dis. 2021;16(1):120.

63. Fuerboeter M, Boettcher J, Barkmann C, Zapf H, Nazarian R, Wiegand-Grefe S, et al. Quality of life and mental health of children with rare congenital surgical diseases and their parents during the COVID-19 pandemic. Orphanet J Rare Dis. 2021;16(1):498-.

\section{Figures}




\begin{tabular}{|c|c|c|c|c|c|c|c|}
\hline Postnatal age & 1 month & 3 months & 6 months & 1 year & $3-4$ years & $7-8$ years & 15 years \\
\hline \multicolumn{8}{|l|}{ Follow-up visits } \\
\hline Pediatric surgeon & $x$ & $x$ & $x$ & $x$ & $\mathrm{x}$ & $x$ & $x$ \\
\hline Pediatrician ${ }^{3}$ & & $\mathrm{x}$ & & $\mathrm{x}$ & & & $\mathrm{x}$ \\
\hline $\begin{array}{l}\text { Dietician/Pediatric Nurse } \\
\text { specialized in nutrition }\end{array}$ & & $\mathrm{x}$ & & $x$ & & & $\mathrm{x}$ \\
\hline Speech therapist & & & & $x^{b}$ & & & \\
\hline \multicolumn{8}{|l|}{$\begin{array}{l}\text { Anthropometric } \\
\text { measurement of growth }\end{array}$} \\
\hline Weight & $\mathrm{x}$ & $\mathrm{x}$ & $\mathrm{x}$ & $\mathrm{x}$ & $\mathrm{x}$ & $\mathrm{x}$ & $\mathrm{x}$ \\
\hline Length & $x$ & $x$ & $x$ & $x$ & $x$ & $\mathrm{x}$ & $x$ \\
\hline \multicolumn{8}{|l|}{ Examination of esophagus } \\
\hline Gastroscopy with biopsy & & & & $\mathrm{x}$ & & & $\mathrm{x}$ \\
\hline $\begin{array}{r}\text { 24-Hour pH-Impedance } \\
\text { measurement }\end{array}$ & & & & $x$ & & & $x$ \\
\hline \multicolumn{8}{|l|}{ Examination of airways } \\
\hline Spirometry & & & & & & & $\mathrm{x}$ \\
\hline Exercise bicycle test & & & & & & & $x$ \\
\hline
\end{tabular}

${ }^{a}$ Voluntary referral to gastroenterologist and pulmonologist

${ }^{b}$ All infants meet a speech therapist before $<1$ year of age and if needed receive additional diagnostics and treatment

\section{Figure 1}

Presentation of the Swedish follow-up program at a tertiary pediatric surgical center for children born with esophageal atresia. The visits include a check-up and multidisciplinary monitoring of digestive and airway problems, growth, development of winged-scapula and scoliosis. At 1 and 15 years of age, patients are at a minimum offered physiological examination of the esophagus (e.g. 24-Hour pH-Impedance, gastroscopy with biopsy and at 15 years, test of the airways (e.g. spirometry) and exercise bicycle test. 

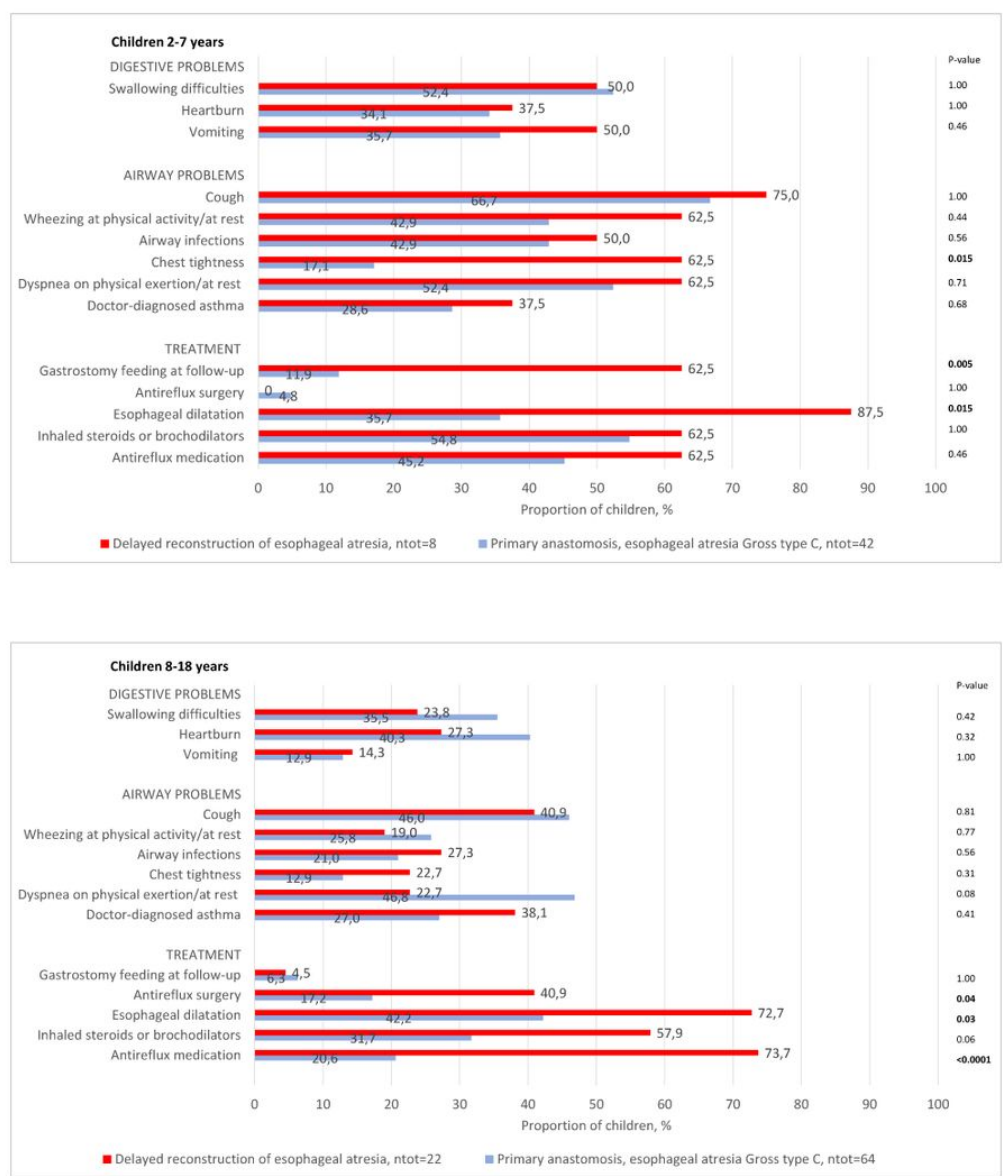

\section{Figure 2}

Symptom prevalence and treatments at follow up in children aged 2-7 (Figure 2a) and in children aged 8-18 (Figure 2b) with delayed reconstruction of esophageal atresia compared to children with esophageal atresia Gross type $\mathrm{C}$ who underwent primary anastomosis. The statistical comparison was performed using Fisher's exact test. Significant level was $p<0.05$. Significant $p$-values are marked with bold text.
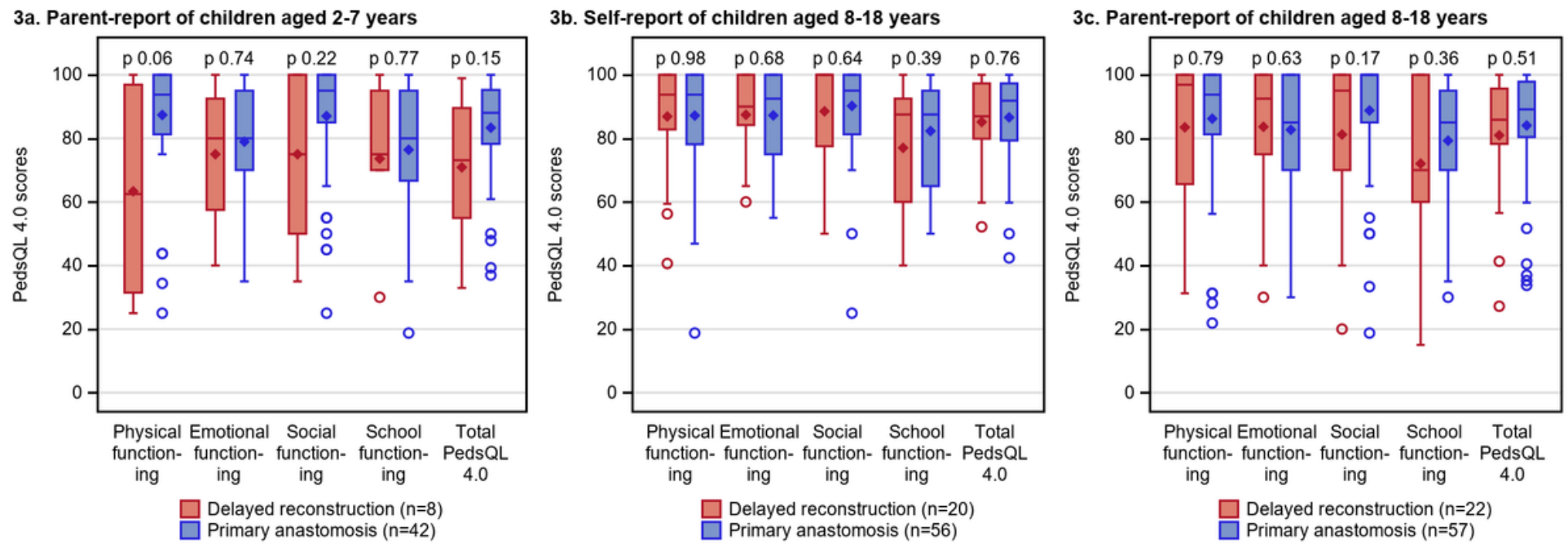

Figure 3 
4a. Self-report of children aged 8-18 years

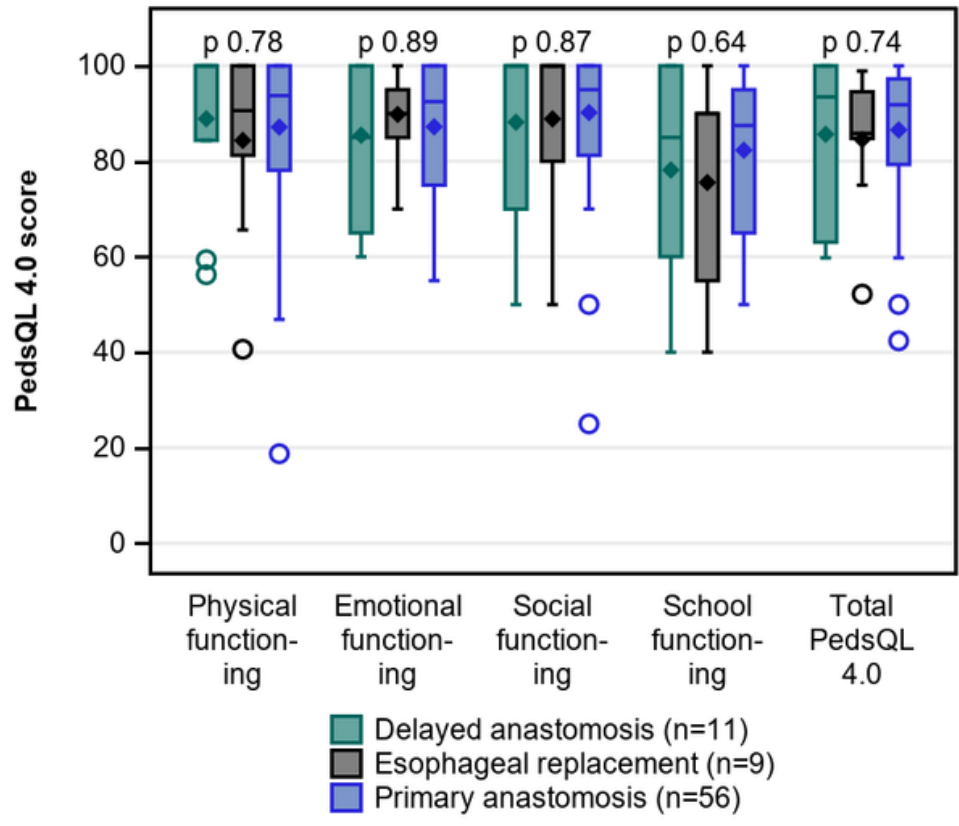

4b. Parent-report of children aged 8-18 years

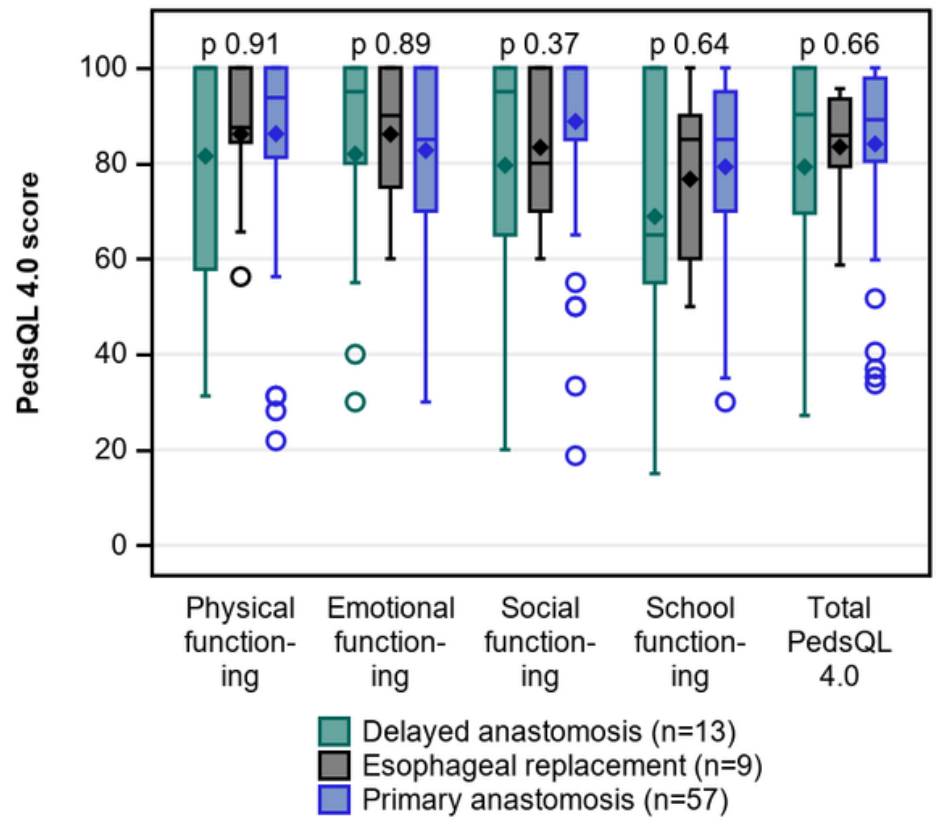

Figure 4

The PedsQL 4.0 scores in children aged 8-18 with delayed primary anastomosis, esophageal replacement and primary anastomosis, self-report (a) and parentreport (b).
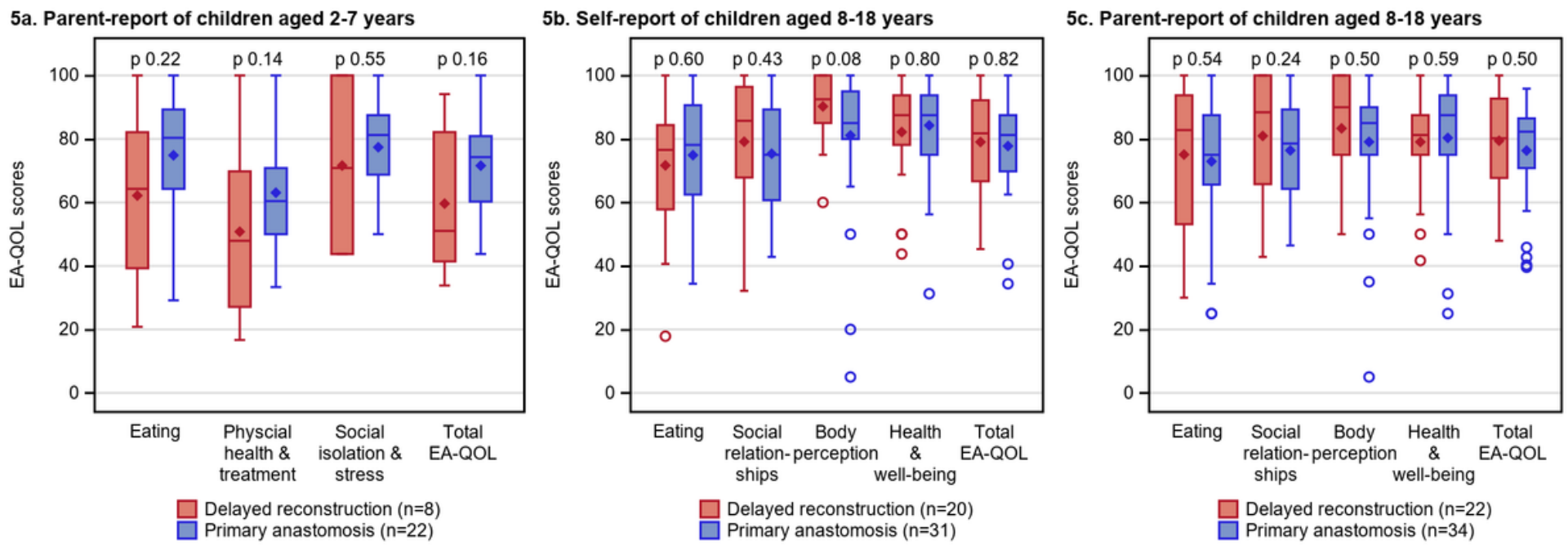

Figure 5

The EA-QOL scores in children aged 2-7 (a) and children aged 8-18 (b-c) with delayed reconstruction of esophageal atresia (including both delayed primary anastomosis and esophageal replacement) compared to children with primary anastomosis of the same age group and gender distribution 
6a. Self-report of children aged 8-18 years

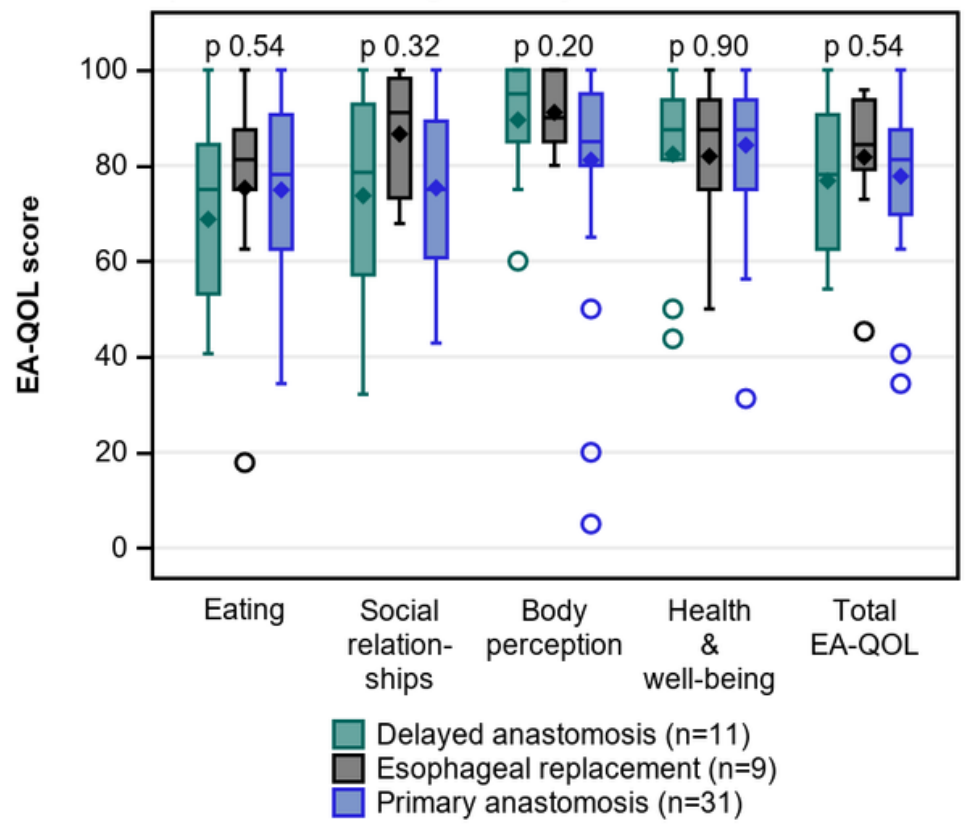

6b. Parent-report of children aged 8-18 years

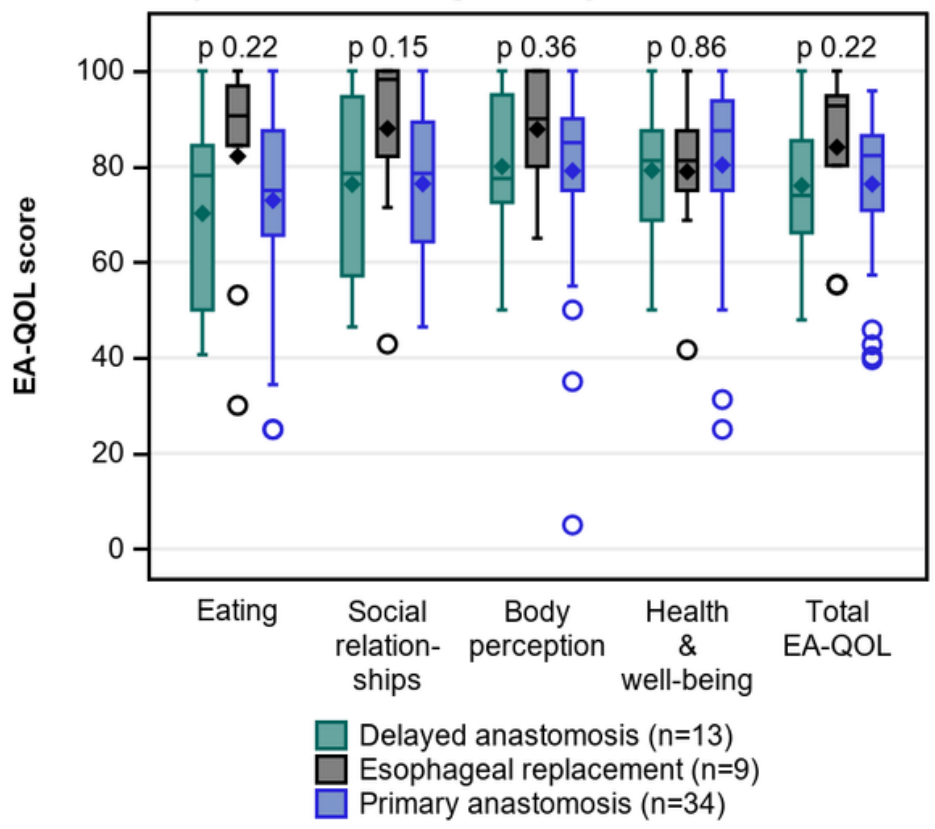

Figure 6

The EA-QOL scores in children aged 8-18 with delayed primary anastomosis, esophageal replacement and primary anastomosis, self-report (a) and parentreport (b).

\section{Supplementary Files}

This is a list of supplementary files associated with this preprint. Click to download.

- Additionalfile1.docx

- Additionalfile2.rtf 